\title{
PRESCHOOL CHILDREN AND EXCESS WEIGHT: THE IMPACT OF A LOW COMPLEXITY INTERVENTION IN PUBLIC DAY CARE CENTERS
}

\author{
Viviane Gabriela Nascimentoㄹ, Janaína Paula Costa da Silva², Thais Costa Machado3, \\ Ciro João Bertoli ${ }^{4}$, Vitor Engrácia Valenti ${ }^{5}$, Claudio Leone ${ }^{6}$
}

\begin{abstract}
Introduction: childhood obesity is a major public health problem, affecting children even at early ages. Objective: to assess the impact of dietary intervention and relatively simple physical activity on the nutritional state of preschoolers. Method: this was an intervention study in public daycare centers targeting children from 2 to 4 years of age, in the State of São Paulo. The sample consisted of 9 daycare centers covering 578 children, with 264 children in the intervention group (IG) and 314 in the comparison group (CG). Intervention was focused on modifications in diet and physical activity, avoiding overloading the routine of daycare centers, for duration of one year. A zBMI score > 1 (zBMI) and $\leq 2$ was considered risk of being overweight, and $>2$ zBMI was considered excess weight (overweight and obese). Analysis was done by frequency calculations, comparisons of proportions by $\chi^{2}$, mean comparisons by t-student and calculations according to Pearson's correlation coefficient. Results: IG showed an inverse correlation between the initial zBMI of the children and the difference with the $z B M I$ at the end of the intervention $(r P=-0.39, p<0.0001)$. The mean difference of $z B M I$ of the overweight children in IG between the beginning and the end of the study period was negative $(-0.46 \mathrm{z}$ score $)$, indicating weight reduction, while the children in the CG was positive $(+0.17 \mathrm{z}$ score) $(p=0.0037)$. Conclusion: intervention in diet and physical activity in overweight preschool children in daycare centers could have a favorable impact on the evolution of their nutritional state.
\end{abstract}

Key words: intervention, obesity, overweight, preschool, child.

\section{INTRODUCTION}

It is a consensus that the presence of obesity is growing significantly among children and that it can result in aggravation of health from childhood to adulthood ${ }^{1}$. The excess of weight in childhood, in regard to its frequency, is an important problem of public health that has been affecting children even at early ages, also in developing countries ${ }^{2}$. An increase in obesity of children and young people has also been verified in Brazili ${ }^{3-6}$.

As a result, an early detection of body mass alterations can be very important, since it can enable early interventions to prevent obesity and possible derived complications. It is worth emphasizing that necessary changes in food habits and physical activities are normally better accepted by younger children, considering that most of these habits are established in this stage of life $^{7}$. With this in mind, innovative educational programs, planned to expand knowledge about children in regard to nutrition and health and also intended to positively exert influence on their diet and reduce sedentary habits, have already been developed ${ }^{8,9}$.

According to Jacob ${ }^{10}$, school programs of health education are, currently, the most efficient strategy to reduce chronic problems of public health resultant from a sedentary lifestyle and an inadequate eating pattern, however more studies in this fields are definitely necessary to reinforce this hypothesis. Taylor et al ${ }^{11}$ demonstrates that it is possible to modify the average Body Mass Index of students between 5 and 12 years of age already after the first year of intervention using a relatively

\footnotetext{
Pos-doctor of the Department of Mother and Child Health of the Public Health School in the University of São Paulo.

2 PhD student of the Department of Mother and Child Health of the Public Health School in the University of São Paulo.

3 PhD student of the Department of Mother and Child Health of the Public Health School in the University of São Paulo.

4 Professor Doctor of the Department of Medicine of the University of Taubaté

$5 \mathrm{PhD}$, Department of Speak and Hearing Pathology, Faculty of Philosophy and Sciences, Paulista State University -FFC/UNESP

6 Professor of the Department of Mother and Child Health of the Public Health School in the University of São Paulo. Corresponding author: leone.claudio@gmail.com
}

Suggested citation: Nascimento VG, et al. Preschool children and excess weight: the impact of a low complexity intervention in public day care centers. Journal of Human Growth and Development 2013, 23(3): 290-295

Manuscript submitted Mai 26 2013, accepted for publication Aug 202013. 
simple intervention of nutritional education, which pointed to a tendency of reduction in excessive weight gain.

Childhood obesity, which is per se a relevant nutritional detour regarding its intensity and frequency, has been increasing not only in high socioeconomic levels of population, but also in populations with lower purchasing power, steadily affecting children at earlier ages. Once installed, the treatment of overweight or obesity is complex and difficult to revert, therefore, early assessment and intervention, either individually or collectively, are important to prevent it to be installed.

Accordingly, in order to prevent overweight and obesity, interventions steadily in earlier ages have been proposed, inclusively in childhood, and there have been stimulation for their assessment. The hereby study has as objective to assess the impact of a pilot project of dietary intervention and physical activity, both of low complexity and simple execution to be performed in public daycare centers.

\section{METHODS}

Observational analytical study of nutritional state of children between 2 and 3 years of age was performed in a sample of 9 public daycare centers, among the 59 existent in the city of Taubaté, São Paulo, Brazil, during the years of 2009 and 2010. These children were the objects of a project that targeted adequacy of caloric offer and promotion of regular physical activity for the preschoolers during the time they stayed at the daycare centers.

Initially, among the almost 2,400 children from 2 to 6 years of age located in these nine daycare centers, 845 of them were identified as having less than four years of age, therefore potentially eligible to be part of the project. Of this total, during the project, 239 children were transferred to other daycare centers and 28 did not receive their parents' and/or responsible persons' authorization to participate, hence only 578 children were re-evaluated by the end of the project out of the initial sample. All children enrolled and attending classes of Maternal I (equivalent to Pre-school in USA) during the years of 2009 and 2010 were included in the project.

Four out of the nine daycare centers were objects of intervention during the school year of 2009 and the other five were not targeted for intervention. During 2010, daycare centers that were not a target of intervention in 2009 became part of the intervention group and the remaining daycare centers no longer took part in the intervention that year. Due to the fact that the intervention was not performed simultaneously in all of the daycare centers of the sample, there were two groups in the end: one of 264 children who were subjected to intervention (named as intervention group - IG) an the other, with 314 children that were not intervention targeted (named as comparison group - CG).
Before the beginning of the intervention, an anthropometric evaluation was performed as part of the pilot project to identify the presence of fortuitous eating disorders. After one year, a new anthropometric evaluation was performed in accordance with the same patterns of the initial one to verify if routine changes have produced any impact over the nutritional state of the children.

By the end of the intervention period, an anthropometric evaluation was also repeated with the CG, also to prevent that a situation was created suggesting any form of discrimination between the children. Weight and height data of this second group, CG, was also analyzed to evaluate their evolution during the same period of time.

As indicative of the intervention impact, score $z$ of Body Mass Index of the children was used to estimate their nutritional state. Accordingly, measuring weight and height were taken in the daycare centers in properly scheduled dates during the months of March of 2010 and 2011 by trained professionals using standardized techniques, in conformity with Lohman et al ${ }^{13}$ description.

All of the children were weighed without shoes and with as little clothing as possible on an electronic scale (SECA ${ }^{\circledR} 803$ ) of capacity up to $150 \mathrm{~kg}$ and subdivisions of $0.1 \mathrm{~kg}$. To measure height, a stadiometer fixed on the wall with subdivisions in centimeters and millimeters was used with children touching their heels, calves, gluteus, and shoulders to the wall and positioning their heads according to the Frankfurt horizontal plane.

Values of Body Mass Index (BMI), calculated by weight and height, were transformed into scores z based on World Health Organization values $2006^{14}$ proposed for each gender and age.

BMI score z (zBMI) criteria proposed in 2008 by the Brazilian Ministry of Health were used to classify nutritional state of children under 5 years of age, which define as risk of being overweight children that present $z B M I>1$ and $\leq 2$, and excess of weight (overweight or obesity) zBMI $>2^{15}$.

The intervention was deliberately elaborated to be of easy application, with the least possible technical complexity, aiming not to change or overload the daycare center routine while its implementation. The project had two components of intervention: the dietary that aimed to adjust the quantity of food offered in the daycare center meals (avoiding inclusively that children take another portion), not modifying food usually offered in the diet of the city schools, the physical activity implemented daily 30-minute physical activities properly adapted for the age of preschoolers. Every intervention was controlled through regular supervision of daycare centers, weekly during the first two months and every other week afterwards ${ }^{12}$.

Based on analysis of databases of the 2 groups (IG and CG), it was possible to estimate the number of children of each group that remained in the daycare centers until the second assessment. For an alpha of $5 \%$, a test power of $80 \%$ was present 
to discriminate a difference of $1 / 4$ of score $z$ of average BMI from the beginning till the end of the intervention period.

The data statistical analysis, within the groups and between the groups, was calculated based on frequencies, comparisons of proportions through the method $\chi^{2}$, comparisons of t-Student means, and calculation of Pearson correlation coefficient.

The number of preschoolers who have changed of daycare center or refused to participate and, as a result, did not complete the intervention was relatively high: $267(31.7 \%)$ children. Therefore, some of the characteristics that these children had presented in the initial evaluation were compared to the characteristics also initially presented of the preschoolers who have completed the intervention.

The project is a result of a partnership between the Department of Education and Culture of the City of Taubaté, the Discipline of Pediatrics of University of Taubaté, and the Department of Maternal and Child Health of Public Health School of the University of São Paulo.

The Research Ethical Committee of University of Taubaté (Protocol nr. 0002/2005) approved the performance of a pilot project of intervention and the Research Ethical Committee of the Public Health School of the University of São Paulo (Protocol nr.
1877/2009) approved the assessment. The Daycare center sent the Terms of Free and Informed Consent to their mothers or responsible person to be properly fulfilled and signed before the collection of anthropometric data started.

\section{RESULTS}

At the beginning of the intervention, 578 children, who afterwards remained in the daycare center for the whole project, presented in average 3.2 years of age. As for genders, they were practically equally distributed, that is, $50.3 \%$ of boys and $49.7 \%$ of girls.

In the initial evaluation of these 578 children, the prevalence of risk of overweight, overweight and obesity totaled $28.9 \%$ (table 1 ). A difference in the prevalence of these nutritional alterations was not observed when younger children, of less than three years of age, was compared to those of who had already completed three years of age or not yet completed four years $(p=0.5882)$.

When children of the two groups, IG and CG, who took part in the whole program, were compared the prevalence of nutritional alterations in the beginning of the intervention with regard to risk of overweight and excess of weight together were (table 1$)$ practically the same $(p=0.46$ and $p=0.42$, respectively).

Table 1: Nutritional classification* of preschoolers of the groups of intervention (IG) and comparison (CG) at the beginning of the intervention. São Paulo, 2010

\begin{tabular}{lrrr} 
Nutritional Status & IG & CG & \multicolumn{1}{c}{ Total } \\
& $\mathbf{n}(\%)$ & $\mathbf{n}(\%)$ & $411(71.1)$ \\
Eutrophic & $185(70.2)$ & $226(72.0)$ & $111(19.2)$ \\
Risk of overweight & $54(20.4)$ & $57(18.1)$ & $35(6.0)$ \\
Overweight & $13(4.9)$ & $22(7.1)$ & $21(3.7)$ \\
Obese & $12(4.5)$ & $9(2.8)$ & $578(100.0)$ \\
Total & $264(100.0)$ & $314(100.0)$ &
\end{tabular}

$\chi^{2}: 2,608, g l: 3, p=0.4561$.

* Ministry of Health, Brazil 2008.

In Figure 1, it is clear that there was an inverse correlation in the intervention group between the initial zBMI of children and its difference

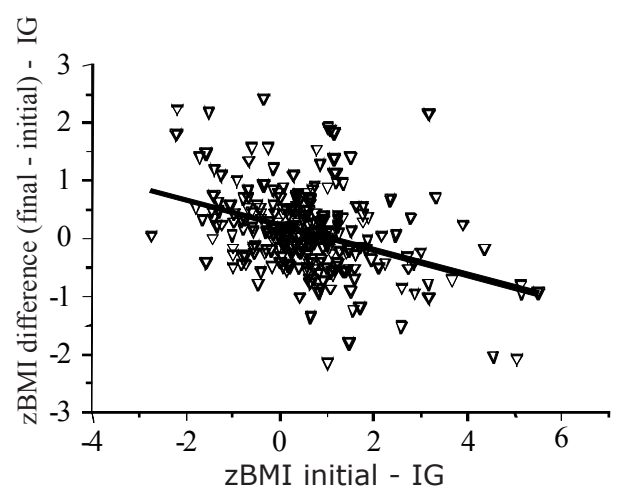

Pearson correlation coefficient: $r=-0.3907$ (IC $95 \%=-0.4884$ to -0.2833 ) $\mathrm{p} \varangle 0.0001 ; \mathrm{R}^{2}=0.0153$ compared to the final zBMI $\left(r_{p}=-0.39\right.$, $\left.p<0.0001\right)$, which was not verified in the group that did not underwent the intervention.

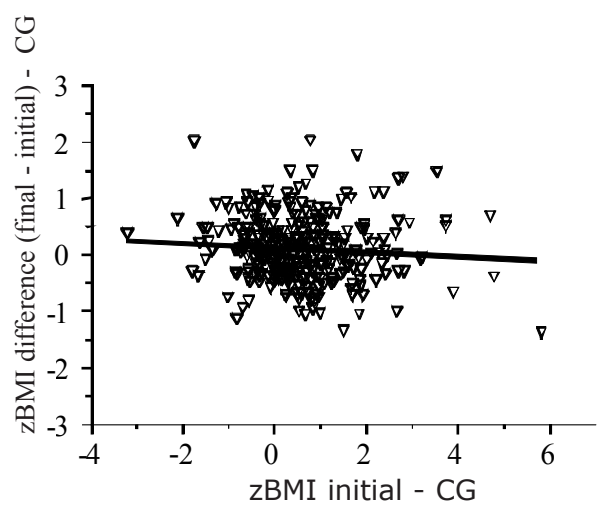

Pearson correlation coefficient: $\mathrm{r}=-0.07940$ (IC $95 \%=-0.1885$ to 0.03159$) \mathrm{p}=0.1605 ; \mathrm{R}^{2}=0.006304$

Figure 1: Distribution of differences of zBMI at the beginning and the end of the study of each child in the intervention group (IG) and the comparison group (CG) according to their initial zBMI. São Paulo, 2010 
Differences of prevalence in these nutritional alterations were also not verified from the beginning until the end of one year when a second anthropometric evaluation was performed (IG: $p=0.27$ and CG: $p=0.25$ ).

In Figure 2, the average differences of zBMI in children of IG with excess of weight (with overweight or obesity) between the end and the beginning of the 12-month period was negative
$(-0.46$ scores $z)$, whereas the average differences in children also with excess of weight of CG was positive $(+0.17$ scores $z)$.

For these children, the differences of zBMI observed between the first and the second anthropometric evaluation (figure 2 ) presented average values statistically different when the groups IG and CG were compared $(p=0.0037)$.

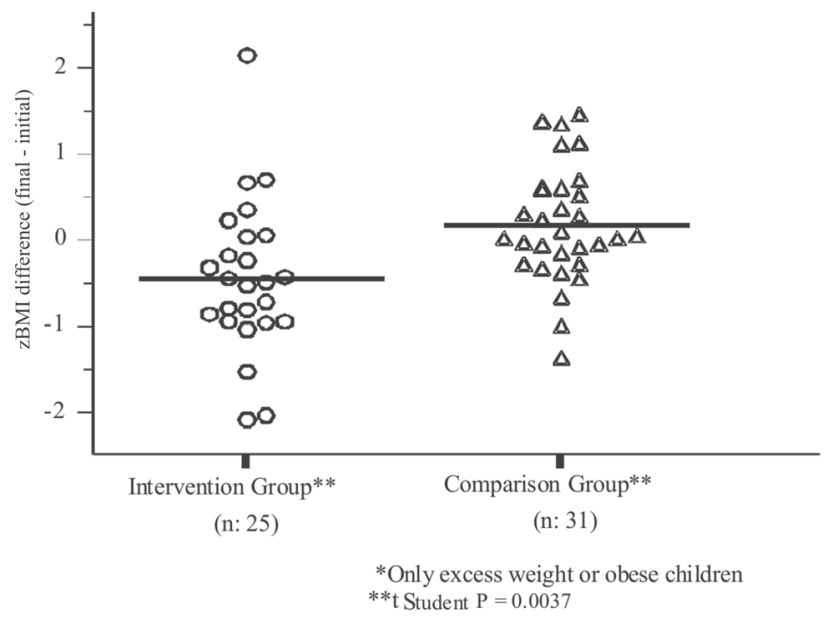

Figure 2: Distribution of differences of zBMI between the beginning and the end of the study of children of the group of intervention (GI) and the comparison group (CG) with excess of weight (overweight + obesity). São Paulo, 2010

In regard to the group of 257 children who did not participate or did not complete the intervention period, it was verified to be very similar to the group that took part in the whole project, since in the comparison of the assessment performed before the beginning of the project there was no statistically significant differences verified in the group for the median of age $(p=0.2595)$, gender distribution $(p=0.2763)$, proportion of children with dietary alterations $(p=0.2595)$, and medians of weight $(p=0.7182)$, height $(p=0.6698)$ and $z B M I(p=0.8432)$.

\section{DISCUSSION}

A low-cost and easy-to-apply intervention in physical activity and diet for one year was not able to reduce the prevalence of excess of weight in a group of preschoolers. In spite of this, at least when the average is considered, it has contributed to a reduction of BMI of overweight or obese preschoolers, that is, for those who already were in a situation of higher risk.

In Brazil nowadays, childhood obesity is already a serious problem for current and, probably, future health of individuals, therefore, early interventions in childhood to prevent obesity can be more rational and less expensive ways to contribute for reducing the incidence of chronicdegenerative diseases.
Considering a population from the nutritional point-of-view, regardless their socioeconomic level, and in accordance with the criteria of Food and Nutrition Surveillance System (Sistema de Vigilância Alimentar e Nutricional) - (SISVAN) of Ministry of Health of Brazil ${ }^{15}$, a proportion of $15.9 \%$ would be expected of normal individuals with zBMI above +1 . The prevalence of risk of overweight and excess of weight in preschoolers herein studied was high, even surpassing in 9.9 and 16.1 points percent of which was expected for, respectively, the intervention groups and comparison groups. These prevalences are even more relevant if a sample of less than 4year-old preschoolers is considered, which suggests a very early beginning of fat accumulation process, possibly even before their admission to daycare centers. This hypothesis is even more consistent when is verified that the prevalence of excess of weight is no different between the groups of children younger than 3 years of age and the group of those of 3 years of age.

This high prevalence in public daycare centers confirms that both risk of overweight and excess of weight is increasingly affecting children at earlier ages and of lower purchasing power, which indicates, in our branch, the necessity to begin interventions even earlier in every socioeconomic level ${ }^{17}$.

The trend to present a lower zBMI at the end of the intervention the higher it was at the beginning is emphasized by the inverse correlation between the initial zBMI and the difference between the final 
zBMI and the zBMI presented at the beginning of the period. This also shows that an intervention easy to be applied, with calorie ingestion capable to be adapted, associated with regular practice of physical activity, and that consequently increases the daily calorie burn of children in daycare centers or preschools, can contribute to improve nutritional state of children between 2 and 4 years of age who already have overweight or obesity.

This fact related above is evidenced when the evolution of the BMI score $z$ of these children is compared to those of the group of children who were not exposed to intervention and that remained in the daycare routine conditions. The fact that both groups (intervention and comparison) come from the same region, are of a similar socioeconomic level and attend daycare centers attached to the same administrative unit ratify, the probability that the benefit observed in fatter children were truly a result of the intervention.

Taylor et $\mathrm{al}^{11}$, after introducing a healthier diet and physical activity for children from 5 to 12 years, also showed that there was no difference in prevalence of excess of weight, nevertheless, there was a significant reduction in the mean zBMI of the group of children who underwent the intervention, thus highlighting that interventions easy to apply and not of extreme complexity can contribute in a not very long period of time to improve the nutritional condition of children.

Systematic review of literature prove that already performed interventions are normally inclined to induce changes in dietary behavior and they are efficient, however, there is no proof of its efficiency in anthropometric indicators of obesity, since most of these interventions are performed as educational lectures and activities about healthy food $^{18}$.

In Brazil, most of the intervention studies performed also have as objective to prevent obesity, which means, they describe interventions focused on activities of nutritional education and of incentive of physical activity practices or, in some cases, only of changes in dietary behavior. Nonetheless, these studies, aside from generally being applied for a very short time, they frequently focus on school students and/or teenagers, which hinder comparisons with the present pilot project ${ }^{19-21}$.

Another complication to establish comparisons with these studies is that they do not associate physical activity with food habits and focus only in food habits in order to prevent obesity. These studies rarely intervene directly in the routine of institutions and hardly produce or evaluate objective results in regard to the children nutritional state, aside from usually reporting short-term, almost punctual, interventions ${ }^{22}$.

The school environment seems to be the only point in common with these intervention studies, showing that school is really an important place to perform a works of intervention. Schools, in addition to being a place intended for education, enable to achieve a large number of children concentrated in the same place and time. Specifically in regard to food, the fact that children have one or more meals per day at schools also helps and certainly propitiates a proper environment to perform a regular work of nutritional education.

Among possible limitations of this evaluation, there is the amount of children lost because of daycare transference and also relative homogeneity of the population who attend these daycare centers. In regard to these losses, initial data of preschoolers who did not complete the period of study were compared with the final sample, it was verified that they were very similar. This result reinforce the homogeneity issue on one hand, however, on the other hand, practically eliminates the possibility of important biases to be introduced in the results because of the losses.

In regard to homogeneity of the population sample, it is worth emphasizing that in certain circumstances it is practically impossible to obtain a really representative sample of the whole universe of children, which would hinder a priori any generalization of results and conclusions. Notwithstanding, as for the pilot project hereby, considering that it is a representative sample of children of urban-population and average-size city with high Human Development Index (HDI), it is possible to presuppose that the results had validity for other urban regions with similar characteristics.

Therefore, as final data interpretation, it is possible to conclude that the type of intervention herein evaluated, which was relatively easy to apply, without great complexity, and low cost, could lead to reduce the risk of very young children to worsen their excess of weight and, possibly, if it was maintained for longer periods, to prevent obesity.

Consequently, it is possible to suppose that in urban populations, very frequent in emerging countries, this type of intervention can substantially contribute with public policies for prevention or contention of the currently observed epidemic of excess of weight. Nor is it less important to bear in mind that, aside from these medium and long-term benefits, the intervention is also able to directly benefit children already affected by the process of nutritional transition that these communities are going through. 


\section{REFERENCES}

1. Meyer F, Mello ED, Luft VC. Obesidade infantil: como podemos ser eficazes? J Pediatr. 2004,80(3):173-82.

2. Chinn S, Rona RJ. Prevalence and trends in overweight and obesity in three cross-sectional studies of British children. BMJ. 2001,322 24-6.

3. Monteiro CA, Conde WL. Tendência secular da desnutrição e da obesidade na infância na cidade de São Paulo (1974-1996). Rev Saúde Pública. 2000,34 Suppl 6:52-61.

4. Abrantes MM, Lamounier JA, Colosimo EA. Prevalência de sobrepeso e obesidade em crianças e adolescentes das Regiões Sudeste e Nordeste do Brazil. J Pediatr. 2002, 78(4): 335-40.

5. Anjos LA, Castro IRR, Engstrom EM, Azevedo AMF. Crescimento e estado nutricional em amostra probabilística de escolares no Município do Rio de Janeiro, 1999. Cad Saúde Pública. 2003,19 Suppl 1:171-9.

6. Schoeps DO, Abreu LC, Valenti VE, Nascimento VG, Oliveira AG, Gallo PR et al. Nutritional status of pre-school children from low income families. Nutrition Journal. 2011,10:43-48.

7. Dietz WH. Childhood weight affects adult morbidity and mortality. J Nutr.1998,128(2):411S-414S.

8. Gortmaker SL, Cheung LW, Peterson $\mathrm{KE}$, Chomitz G, Cradle JH, Dart $\mathrm{H}$ et al. Impact of a school-based interdisciplinary intervention on diet and physical activity among urban primary school children: eat well and keep moving. Arch Pediatr Adolesc Med. 1999,153(9):975-83.

9. Sahota $P$, Rudolf MCJ, Dixey R, Hill AJ, Barth $\mathrm{JH}$, Cade J. Evaluation of implementation and effect of primary school based intervention to reduce risk factors for obesity. BMJ. 2011,323:1-4.

10. Jacob A. School programs. In: Chen C, Dietz WH. Obesity in Childhood and Adolescence. Philadelphia: Lippincott Willians \& Wilkins, 2002. p. 257-72.

11. Taylor RW, McAuley KA, Barbezat W, Strong A, Williams SM, Mann JI. APPLE Project: 2-y findings of a community-based obesity prevention program in primary school-age children. Am J Clin Nutr. 2007, 86(3): 735-42.
12. Nascimento VG, Salvador EP, Silva JPC, Bertoli CJ, Blake MT, Leone C. Overweight in preschool children: analysis of a possible intervention. J Hum Growth Develop. 2012, 22(1):11-16.

13. Lohman TG, Roche AF, Matorell R. Anthropometric standardization reference manual. Illinois: Human Kinetics Publishers, 1998.

14. World Health Organization. Child growth standards based on length/height, weight and age. Acta Paediatr. 2006, 450:76-85.

15. Ministério da Saúde. Departamento de atenção básica. Sistema de Vigilância Alimentar e Nutricional. http://nutricao.saude.gov.br \sisvan.php?conteudo =curvas_cresc_oms Acessado em 01/08/2009.

16. Onis $M$ de, Lobstein $T$. Defining obesity risk status in the general childhood population: which cut-offs should we use? Int J Pediatr Obes. 2010,5:458-60.

17. Nascimento VG, Bertoli CJ, Bertoli LMQ, Feferbaum R, Abreu LCA, Leone C. Secular trend of growth of preschool, Brazil. Journal of Human Growth and Development. 2010, 22:199-207.

18. Van Cauwenberghe E, Maes L, Spittaels H, van Lenthe FJ, Brug J, Oppert JM et al. Effectiveness of school-based interventions in Europe to promote healthy nutrition in children and adolescents: systematic review of published and 'grey' literature. $\mathrm{Br} J$ Nutr. 2010,103(6): 781-97.

19. Vargas ICS, Sichieri R, Sandre-Pereira G, Veiga GV. Avaliação de programa de prevenção de obesidade em adolescentes de escolas públicas. Rev Saúde Pública. 2011, 45(1):59-68.

20. Kain J, Concha F, Salazar G, Leyton B, Rodríguez $M P$, Ceballos $X$ et al. Prevención de obesidad en preescolares y escolares de escuelas Municipales de una Comuna de Santiago de Chile: proyecto piloto 2006. Arch Latinoamer Nutr. 2006,59(2):139-46.

21. Gabriel CG, Santos MV, Vasconcelos FAG. Avaliação de um programa para promoção de hábitos alimentares saudáveis em escolares de Florianópolis, Santa Catarina, Brazil. Rev Bras Saúde Matern Infant. 2008,8(3):299-308.

22. Pérez-Morales ME, Bacardí-Gascón M, JiménezCruz A, Armendáriz-Anguiano A. Intervenciones aleatorias controladas basadas en las escuelas para prevenir la obesidad infantil: revisión sistemática de 2006 a 2009. Arch Latinoamer Nutr. 2009,59(3):253-59. 